Proceedings of the 2010 Winter Simulation Conference

B. Johansson, S. Jain, J. Montoya-Torres, J. Hugan, and E. Yücesan, eds.

\title{
AN EXPERIMENTAL DESIGN AND PRELIMINARY RESULTS FOR A CULTURAL TRAINING SYSTEM SIMULATION
}

\author{
Paul A. Fishwick \\ CISE Department \\ Bldg. CSE, Room 301 \\ University of Florida \\ Gainesville, Florida 32611, USA
}

Amy Jo Coffey

Journalism and Communications

Weimer Hall, Room 2042

University of Florida

Gainesville, Florida 32611, USA

\author{
Rasha Kamhawi \\ Journalism and Communications \\ Weimer Hall, Room 2040 \\ University of Florida \\ Gainesville, Florida 32611, USA
}

Julie Henderson

P. K. Yonge Research Developmental School

1080 SW $11^{\text {th }}$ Street

University of Florida

Gainesville, Florida 32601, USA

\begin{abstract}
Computer simulation has been widely deployed by the military for force-on-force based training but only more recently for training researchers, analysts, and war-fighters in matters of cross cultural sensitivity. This latter type of training gives the trainee a sense of "being inside" a target culture. We built the Second China Project as a hybrid immersive, knowledge-based software platform for use in cultural training. Is this training effective? More specifically, what are the effects of immersion on memory and other cognitive variables? We chose to base our research questions, not around a specific user group, but more generally around a category of training system--one involving the use of multi-user virtual environments (MUVEs). We present the architecture of an experiment designed to test whether MUVEs are effective training platforms, and to explain the process used in developing a testing environment to determine the precise nature of that effectiveness. We also discuss lessons learned from the earlier pilot study and ongoing experiment.
\end{abstract}

\section{INTRODUCTION}

Simulation involves the creation and analysis of models, including key aspects such as verification and validation. Models must capture an aspect of the physical world and then through analysis, we assess that model. Typically, this assessment is in the form of verification (i.e., comparing the model with the software implementation and the requirements) and validation (i.e., comparing model inputs and the output response to the physical response). For a particular class of model with objectives of education or training, our assessment is based on human testing. With the increase in the use of enhanced human-computer interfaces for computer simulation, we become as concerned with the effect of the simulation components on a human subject as we are with the accuracy of a model in its partial replication of physical behavior. This focus--human performance--extends simulation analysis beyond verification and validation into a broader category that becomes central to the simulation field, using methods of science to form the tool set. The term "human performance" is used to capture behavioral and cognitive effect. 


\section{Fishwick, Kamhawi, Coffey and Henderson}

Our research is grounded in the Second China Project (Fishwick et al. 2008, Henderson et al. 2008, SC 2010). Second China is implemented in Second Life (Weber et al. 2008). This project has two goals: (1) to construct an educational and training simulation environment for Chinese culture by using a linked Web/MUVE, and (2) to assess the simulation environment by measuring human performance. The first goal was achieved during 2009 and the second goal is ongoing with about 21 human subjects out of a total of 160 remaining to process.

We focus on the second goal by describing the experimental design and implementation and then summarizing key results to date taken from the pilot study and the ongoing experiments. The paper is not meant to reflect definitive findings since the study is not yet complete. Instead the objective is to describe a simulation-based engineering design and implementation required for assessment to occur. The paper is organized as follows: the overview of the experiment in terms of design and implementation is covered in Section 2, followed by a description of the instruments used for measurement in Section 3, and initial results to date in Section 4. Section 5 covers work related to ours, and we conclude in Section 6.

\section{EXPERIMENTS}

Our human subject study was based on the first phase of the Second China project where we constructed an MUVE and a web-based knowledge set of learning modules about Chinese culture. Several snapshots of the MUVE are shown in Figure 1, and snapshots of the web-based learning modules are shown in Figure 2.
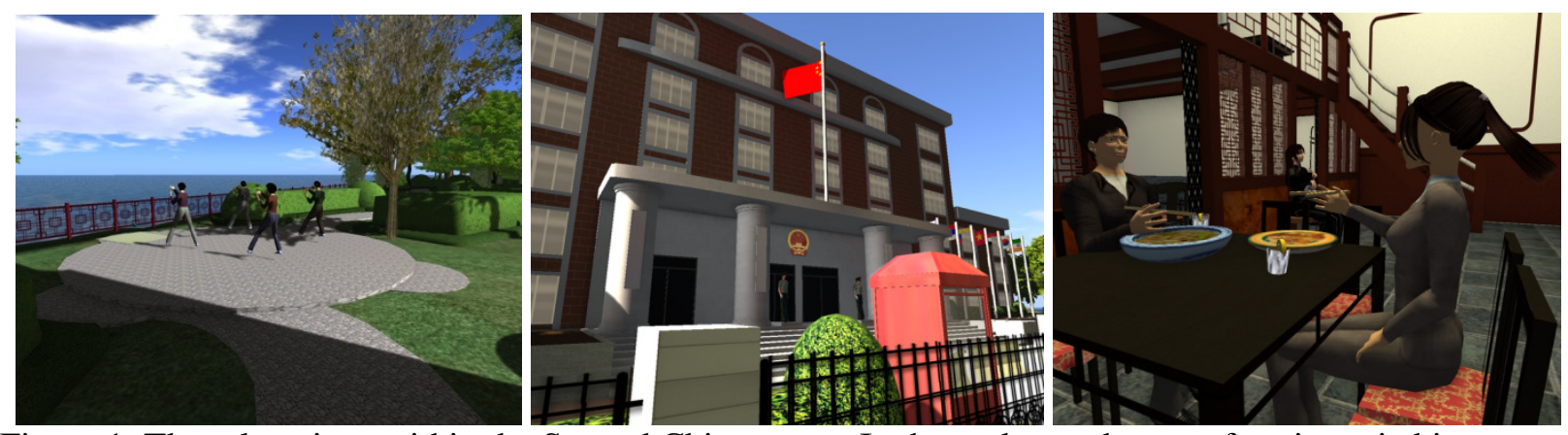

Figure 1: Three locations within the Second China space: In the park area-bots performing tai chi, government building across from the square, and inside the tea house. From (SC 2010).
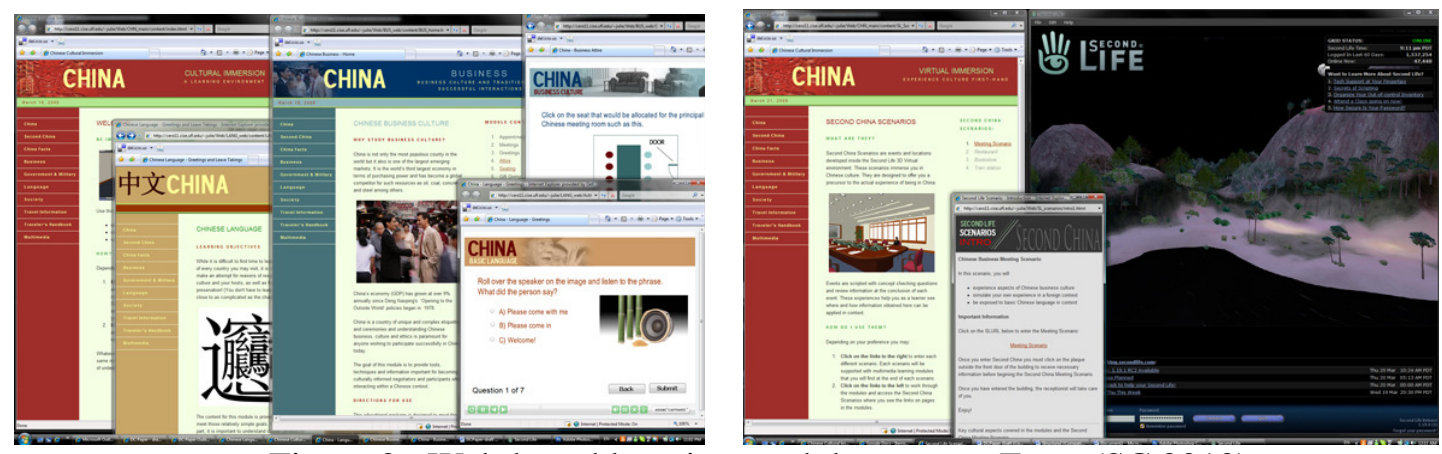

Figure 2: Web-based learning module content. From (SC 2010).

When studying humans, there are a number of procedural actions that must take place, including the approval of an Institutional Review Board (IRB) at the start of the study followed by IRB application renewals if the study parameters change or re-application is necessitated due to time extensions. The beginning of any study is to derive the research questions (RQs). At first, this may seem to be a pedestrian ac- 
tivity, but it is indeed challenging and it drives the sort of study to be performed. Informally, our interest could be defined as the following primary research question:

RQ: What are the benefits on the trainee of an MUVE compared with simpler, and more inexpensive, training solutions?

When we originally asked this question, we recognized that there was significant interest in MUVEs across the education communities, but that little work had been done in formally evaluating the touted benefits of MUVEs. Are they really effective, and if so, how? One also needs to determine the social or behavioral science methods needed to answer this question. Various options exist and they are not mutually exclusive: poll, survey, focus group, in-depth interview, content analysis, and experiment. We chose an experiment since that was deemed to be able to provide the most accurate answer to our question. Experiments are concerned with people's responses to treatments under controlled circumstances. A treatment is the condition to which the subjects are exposed. A control means that one attempts to isolate the effects of the treatment by making sure that all other factors are held constant. Our treatment would consist of different levels of the variables of interest to determine their effect on cultural sensitivity and cognition. The MUVE (Figure 1) was used in the experiment and the web-based learning modules (Figure 2) were used as raw material from which to generate the cultural questions for the questionnaires.

Typically, there are many different measurements for humans that one can use, depending on the goals and research questions. For example, this is a partial list: 1) attention: how long (and where) is the subject most attentive to stimuli?, 2) memory: what is the level of recognition, cued recall, and free recall?, 3) emotion: arousal and valence, 4) attitudinal change, 5) behavioral change, and 6) self-reports (subjective). These represent cognitive measures; physiological measures are also possible and are planned, but not reported in our study. For example, skin conductance and heart rate are indices of valence, attention, and arousal. Both subjective (Kamhawi and Grabe 2008) and physiological measures represent valid options when studying human subjects.

\subsection{Population}

It is necessary to define the population of interest when conducting an experiment. Our population was undergraduate university students at the University of Florida. While this population is not a specific match with the ultimately envisioned end users of the study (e.g., analysts, war-fighters, linguists), there was no reason to believe that young adults and this military population would respond differently, both cognitively and emotionally, when exposed to a foreign culture with which they have had no prior experience. In addition, and from a more pragmatic standpoint, for the experiment to take place, we required sampling from a larger and more accessible population and the university population was a logical choice. Subjects are filtered as being acceptable or unacceptable prior to their data being processed for analysis. Subjects that are "filtered out" are in one of three categories: (1) Chinese ethnicity or familiarity with this culture- a subject may have a family or socio-cultural background which could serve as a bias (e.g., the subject may know answers to questions without experiencing the stimuli), (2) knowledge of experiments the subject may know how experiments are conducted and might guess at reasons for specific questions, thus potentially causing a bias, and (3) advanced technical knowledge - the subject may be technologically advanced with regard to multi-user virtual environments or coding bots, and this might lead the subject to dwell on technical issues rather than focusing on answering the cultural questions. Recruiting of subjects is performed across the university population, seeking to recruit in those classes not reflective of the previously defined three categories. Subjects are offered \$20 or extra credit, with the instructor's consent. Participation is entirely voluntary. 


\subsection{Refined RQs Yielding Two Experiments}

The research question RQ, although a necessary first step, had to be refined into more concrete questions that could be tested. We created the following two questions:

RQ1: What effect does sense of presence have on cultural memory and intercultural sensitivity?

RQ2: How does an immersive environment compare and contrast with a--presumably--less immersive environment such as the Web?

RQ1 and RQ2 provided us with a base from which to design two experiments: one to investigate the role of presence in terms of fundamental differences in amounts of immersion and interaction, and the other to conduct a channel experiment, exposing subjects to one of two channels: immersive (i.e., Second China) and non-immersive (i.e., flash-based animation with audio narration on a web page). Subjects were randomly assigned to one of eight orders, with 4 orders associated with Second China and the remaining 4 with the web stimulus.

For experiments, it is important to use several messages that share a particular factor rather than test the effects of the factor from one message. Since SL stimuli are complex ones involving many variables, there can be several confounding factors that need to be controlled. These are controlled by randomizing them across a range of stimulus messages (Geiger \& Newhagen, 1994). We had two scenarios almost ready for use, and two others were created for a total of four scenarios to serve as the stimuli. The topics of the four scenarios were a Chinese business situation, a visit to a Chinese tea house, Chinese government and politics, and the fourth discussed Chinese parks, streets and money matters. Both experiments used these four culturally authentic scenarios as the basis for generating the stimuli.. We also created four orders to remove any bias that could be caused through the order of scenario exposure having a potential effect on the subject. An order was defined by a sequence of scenario exposures. For example, one order could be 2, 1, 3, 4 indicating the index of the scenario to be run for subjects falling into that order.

The length of time necessary for the participant to complete the activity are approximately 2-2.5 hours for Experiment 1, and 1.5-2 hours for Experiment 2. Delayed measures take 30 minutes, yielding a maximal time per subject as 3 hours for the Second Life subjects and 2.5 hours for the Web subjects.

\subsection{Experiment 1: Sense of Presence (RQ1)}

To study the role of sense of presence, we chose the following independent variables: interaction, immersion, order, gender, and expertise. A low level of interaction was defined by the subject not being allowed to use an input device except at the first and last event as part of a scenario, with the first input triggering the start of the scenario and the last input resulting in the user's avatar sitting down. A high level of interaction was defined by the user interacting throughout the scenario using the mouse and keyboard. A low level of immersion was an over-the-shoulder view of the avatar interacting with the environment, whereas a high level of immersion was a head-based reference frame indicating a view through the avatar's eyes. Each scenario was created in four different versions, with different combinations of interaction and immersion: one low interaction and low immersion, one high interaction and high immersion, one low interaction and high immersion and one high interaction and low immersion. Each participant was exposed to the four different topics/scenarios, presented at different levels of immersion and interaction, so that each participant was exposed to one scenario in high immersion and high interaction, another scenario in high immersion and low interaction, a third scenario in low immersion and low interaction and another scenario in low immersion and high interaction. Order was a control variable to eliminate the effects of the order of presentation. Gender consisted of two levels, male participants and female participants. Expertise is defined as whether an individual has a certain level of 3D game-playing proficiency or MUVE experience 


\section{Fishwick, Kamhawi, Coffey and Henderson}

(i.e., with expert and non-expert levels). There were four orders, varying the sequence of scenarios used for each subject. Subjects always started with an acclimation scenario, allowing them to become accustomed to the MUVE technology, prior to beginning the first sequence of that order.

The variables of immersion and interaction are within-subjects variables since each subject experiences each level of the variable. The remaining independent variables of order, gender and expertise are termed between-subjects variables because a subject can be only one level of the variable and not the other. For example, the subject has no choice of gender or level of expertise. Dependent variables for experiment 1 included: Sense of presence (SOP), subjective evaluation measures, cued recall and recognition, and cultural sensitivity. Dependent and independent variables are listed in more detail in appendices A and $\mathrm{B}$ for this experiment and the following one.

\subsection{Experiment 2: Channel (RQ2)}

The second experiment was to study human cognitive performance related to a different channel--one that had no immersion or interaction, only flash-based narrated sound slide presentations embedded in a web page. The same four scenarios were used as for Experiment 1. Independent variables were channel, order, gender, and expertise. These are between-subjects variables. Dependent variables were the same as the dependent variables for Experiment 1.

\subsection{Scenarios and Orders}

During the first phase of our project, our goal was to build scenarios for purposes of training an individual on Chinese culture, however, it became apparent that each scenario differed in types and quantity of cultural exposure. It was necessary to control all these scenario differences so we can test the effects of immersion and interaction on our dependent variables and eliminate the possibility for contaminating these results by other differences in the scenarios. To guard against the possibility of these differences resulting in different, and unintended, outcomes in the experiment, we need to operationalize the environment so that all scenarios were equal in terms of quantity of cultural information, level of interest to users, and number of bots visible to the user. For example, each scenario had exactly five units of cultural information presented by audio to the subject. Navigation was regulated by placing red dots as waypoints indicating where the user was to make his/her next move. Having multiple orders within the experiment served a similar purpose--to remove the possibility of contamination or bias resulting from the way the scenarios were ordered while maintaining the essence of the original, and training-motivated, culturally-rich scenarios. In the design of the stimuli there is a dilemma where the researcher has to choose a point between two extremes on a continuum, control and regulation of all aspects of the environment and less control to keep the true nature of the environment. The first is necessary to remove all possibilities for any extraneous variables that can affect how the users react to the medium. The environment could be "sterilized" of all extraneous variables to reduce the chance of error in the results; the effect studied is that of the independent variables and nothing else. The second is necessary to claim ecological validity, to keep the extraneous elements of the environment that make it unique or different from other media. In this experiment, for example, the researchers used red buttons to guide the users into taking certain routes within the environment, a form of control that is not present in the non-experimental, education-oriented version of this medium, but necessary to ensure that all users were exposed to exactly the same pieces of information that were also presented to the Web group. At the same time variables that are true to the virtual environment--such as the presence of other beings (bots) in the space and allowing the users to freely navigate from one scenario location to another--were added to make the environment as faithful as possible to the non-experimental version of the virtual environment.

Figure 3 shows a top view of a sample scenario: the Tour Scenario, where the subject learns about Chinese government. The cultural content is triggered by or related to items encountered in the MUVE, thereby contextualizing cultural content as it may be encountered or triggered in real life. For example: 
the Chinese Communist Party (CCP) emblem triggers information about the CCP and the US flag triggers information about U.S.-China relations. This is typical of each of the four scenarios. The subject starts at a "red X" near a bot which serves as a walking guide for the scenario. The yellow path shows the path taken by both the bot and the subject, who follows the bot along the path. The subject begins at location $(29,69.24)$ which is an X, Y, Z location, and then walks by the government building and then moves toward and sits on a park bench located in the government square across from the building. The bot guides the user's avatar and, while speaking, provides cultural information by having the subject interact with (in the case of high interaction) or look at (in the case of low interaction) a monument and a globe.

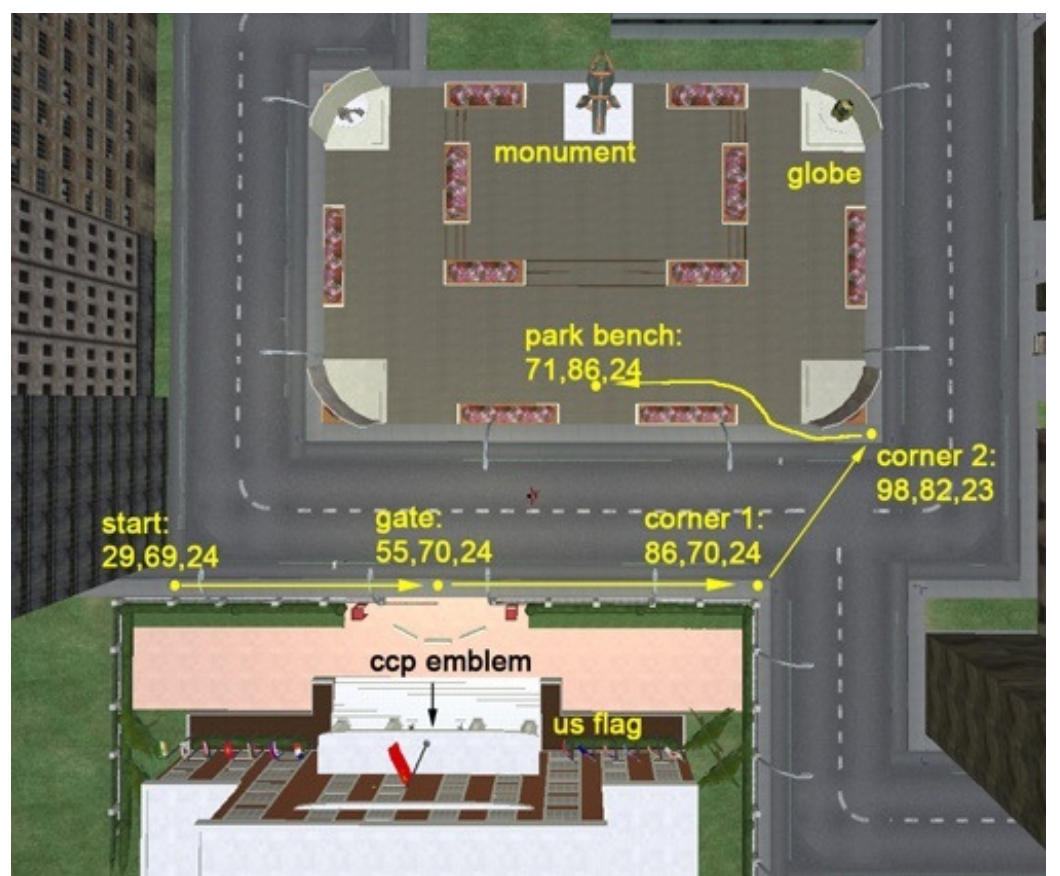

Figure 3: The government scenario -- one of four on the island.

Table 1 illustrates a part of the finite state machine (FSM) used to formally define the behavior of the bot in each scenario and the cultural content to be delivered, and thus, the order of individual scenario events. The FSM was useful in not only defining interactive bot behavior, but also served as a means of communication among team members responsible for the definition of culturally appropriate actions and software engineers who were required to write code to facilitate these actions within Second Life. For example, the start state of the FSM indicates where the bot is to be located in X,Y,Z coordinates $(29,69,24)$ and when the government scenario begins, audio is delivered which states "Click on the red button." The event of the avatar touching the red button causes a state transition where the bot says, "Please come with me." Events that cause change of state tend to be either touch or proximity events. 
Table 1: A subset of the finite state machine used in the government scenario.

\begin{tabular}{|c|c|c|c|}
\hline STATE & ACTION/OUTPUT & EVENT/INPUT & NEXT_STATE \\
\hline $\begin{array}{l}\text { START } \\
\text { Corner of } \\
\text { Zhongshan and } \\
\text { Jianguo Roads } \\
\text { Second China } \\
29,69,24\end{array}$ & $\begin{array}{l}\text { Say(Instr, Click on the red } \\
\text { button on your guide in order to } \\
\text { tell her that you are ready to } \\
\text { take the tour.) } \\
\text { Delay(3) } \\
\text { Flash(RedButton, on guide) }\end{array}$ & $\begin{array}{l}\text { Touch } \\
\text { (AV,RedButton_guide) }\end{array}$ & TRIGGERING_GUIDE_1 \\
\hline $\begin{array}{l}\text { TRIGGERING_ } \\
\text { GUIDE_1 }\end{array}$ & $\begin{array}{l}\text { SayAudio(GUIDE, Please come } \\
\text { with me) } \\
\text { Say(Instr, Please follow Ting, } \\
\text { your guide to the gates of the } \\
\text { Government building.) } \\
\text { Delay(3) }\end{array}$ & & FOLLOWING_GUIDE_1 \\
\hline $\begin{array}{l}\text { FOLLOWING_- } \\
\text { GUIDE_1 }\end{array}$ & $\begin{array}{l}\text { Walk(GUIDE, To_Gates) } \\
\text { Walk(AV, To_Gates) }\end{array}$ & Proximity(AV, Guide) & AT_GATE \\
\hline $\begin{array}{l}\text { AT_GATE } \\
55,70,24\end{array}$ & $\begin{array}{l}\text { Say(Instr, Turn to look at the } \\
\text { front of the building. Click on } \\
\text { the red button on the communist } \\
\text { party logo to learn about the } \\
\text { Chinese Communist Party) } \\
\text { Delay(3) } \\
\text { Flash(RedButton, } \\
\text { On_CCPLogo) }\end{array}$ & $\begin{array}{l}\text { Touch(AV, } \\
\text { RedButton_CCPLOGO) }\end{array}$ & $\begin{array}{l}\text { LEARNING_ABOUT_CC } \\
\mathrm{P}\end{array}$ \\
\hline
\end{tabular}

\section{INSTRUMENTS}

The dependent variables, sense of presence and intercultural sensitivity, were measured with specially developed scales. Presence itself refers to "a sense of being there" within the virtual environment (Marsh, Wright, \& Smith, 2001) and is a "key aspect of the virtual experience" (Waterworth \& Waterworth, 2001, p. 211). Sense of presence has been measured in a multitude of ways (Mikropoulos, 2006), but our research team determined the ITC-Sense of Presence Inventory (ITC-SOPI), developed by Lessiter, Freeman, Keough, and Davidoff (2001), to be best suited for this study. ITC-SOPI measures four identifiable presence types or factors: spatial presence (related to sense of physical presence), engagement (with the objects and others in the stimuli), ecological validity (how natural the environment feels), and negative effects (e.g. experiencing eye strain or feelings nauseated). The ITC-SOPI uses a Likert-type scale, with respondents rating their experiences for each item asked.

For intercultural sensitivity, the Intercultural Sensitivity Scale (ISS) by Chen and Starosta (2000) was used. However, this instrument was modified in order to not only measure intercultural sensitivity toward other cultures in general (intercultural sensitivity), but also sensitivity toward one culture, that of China 


\section{Fishwick, Kamhawi, Coffey and Henderson}

(cultural sensitivity). In order to accomplish both ends, the original ISS was expanded to include a parallel set of scale items inquiring about feelings toward Chinese culture and persons, in addition to the existing instrument that asked about feelings toward other culture and persons in general. The instrument, then, was double in size to accomplish the purposes of this study and to be able to compare and contrast Second China's effect on both (a) cultural sensitivity toward China and its people, and (b) intercultural sensitivity toward other cultures in general.

\section{INITIAL RESULTS}

While final results are still pending, preliminary analysis based on the first 50 subjects reveals some promising findings. We qualitatively summarize them here. For Experiment 1 (Sense of Presence), we were looking for any interaction effect between immersion and interaction, the two elements comprising our operational definition of "sense of presence." We were looking for any statistically significant effect that the combination of these two independent variables, working in tandem, may have had upon our dependent variables of cultural knowledge and cultural sensitivity. We did not find any such interaction. It seems that higher perceived interaction by subjects produces a greater sense of presence on spatial and engagement factors. The greater the perceived interaction level, the more intensely involved they feel, and the more aware they are of the space and objects surrounding them within the virtual space. In addition to the positive outcomes for spatial and engagement presence factors, the perceived high interaction condition (when compared with low interaction) made subjects feel more in control and happier, and they perceived the high interaction condition of the virtual scenario as more believable, and just slightly more informative and objective.

Experiment 2 (Channel), early results suggest that Second Life may be a more effective medium than the Web for increasing cultural learning, at least on some levels. Specifically, visual recognition (immediate and delayed) produced significantly better results within Second Life than within Web stimuli, and showed superior results for all four presence factors-"spatial," "engagement," "ecological validity/naturalness," and "negative effects"- as well as for some of the evaluation measures. Verbal (textbased) recognition did not produce superior results within Second Life. Preliminary results for cultural sensitivity were not analyzed.

Overall, initial results seem promising for the use of 3D virtual environments for increasing cultural learning, particularly those 3D virtual spaces, such as Second China, that provide perceived high interaction conditions, and that provide visually-based learning opportunities. Generally speaking, subjects were happier when using Second Life, and perceived the content in that environment as more important and more enjoyable, than compared to Web scenarios of the same content. It is possible that main effects will yet surface for immersion or that an interaction effect will appear for the combination of immersion and interaction, once all subjects $(N=160)$ have been tested and the study is complete.

\section{RELATED WORK}

Related work is viewed within the simulation community, and also within the study of presence. Within the simulation community, related research on the formation of similar environments to ours includes the following areas: training, education, and agents; however, the most relevant related work is in how these environments are assessed in terms of their effects on humans. Fishwick (2007) provides a compilation of modeling methods, including agent-based approaches and those based on state machines used for coding the bot behaviors for each scenario. Agent-based simulation (Luke et al. 2005; Yilmaz et al. 2006) is particularly suited to this type of scenario dynamics. The use of virtual reality is well known in simulation (Macredie et al. 1996) but is only recently being used within multi-user virtual environments (Fishwick et al. 2008). The general area of verification and validation in simulation (Sargent 2009) covers methods for verification of implementation against requirements and validation of model behavior against observed physical behavior, but is not focused on human performance. Formal studies of humans regarding modeling and simulation are beginning to appear (Tako and Robinson 2009, Monks et al. 2009). In terms of 


\section{Fishwick, Kamhawi, Coffey and Henderson}

empirically analyzing virtual environments, Cooper (2009) finds support for one of two hypotheses indicating a positive relationships between level of engagement and performance. Virtual environments such as Second Life support broad-based educational goals such as multiple perspectives, situated learning, and transfer to real-world environments (Dede 2009).

Work performed in studying the cognitive effects of presence has been conducted in relation to health communication, e.g. in an effort to treat anxiety and phobias (Hodges et al., 1994; Ku et al., 2006; Orman, 2003, 2004; Rothbaum et al., 1995; Schuemie et al., 2001; Slater et al., 2006) and medical students are using virtual environments as training aides for patient interaction (Deladisma et al., 2007; Stevens et al., 2006). The U.S. military has begun exploring the value of virtual environments as a way to relieve combat stress (Etengoff, 2008; Vargas, 2006; Cain Miller, 2009). Commercial entities such as IBM have also experimented with Second Life, to better understand the economic and marketing potential the environment might provide (Holtz, 2007). Reuters and CNN are among the journalism organizations experimenting there, though more studies examining virtual environments' possible applications to mass media and journalistic endeavors are needed.

\section{CONCLUSIONS AND LESSONS LEARNED}

The first half of our Second China work was building the environment --both in terms of all of the cultural knowledge in the web-based learning modules as well as the Second Life-based environment, Second China. Building that environment for learning and training came with its own set of challenges. The second half of our work has been to test this environment, and this manuscript serves as a summary of that experience. At this writing, the experiment has not concluded $(21$ subjects remaining from a total sample of 160), and we estimate completion in July/August 2010. After completion, there will be a vast amount of data which must be analyzed. The following represent some key lessons learned from the experience, with regard to the testing phase of the project. These lessons primarily reflect items that resulted in increased time, labor, and expense:

- Stimuli creation: originally, two scenarios were built. However, for the design of an experiment while varying order of exposure to scenarios, we needed four scenarios. Also, we needed to construct an acclimation scenario for those without the proper requisite background in navigating 3D environments. The key issue, however, was that scenarios that may be appropriate for learning and training are not necessarily ready to be directly employed within an experiment. We placed red buttons to force waypoint navigation and were required to standardize the look and feel of each scenario. To do otherwise would jeopardize the experiment through scenario bias. We also have encountered technical problems with the Second Life service, but mainly in the operation of the bots, which are integral to providing social presence in-world. Unfortunately, bots are not officially supported by Linden Labs, although they are permitted if identified as such and if used for education and training purposes on owned land. Bot clothing and meshes would frequently turn grey, and bots would disappear at random times. Some subject data had to be discarded due to technical problems.

- Cognitive overload: we ran a pilot study prior to the experiment following a peer review and informal focus group feedback. The pilot was particularly useful because we were obtaining results pointing to subjects reporting greater sense of presence with web stimuli than within the MUVE. We hypothesized that this anomaly was due to the use of text in providing all instruction-based and culturally-based material. The text was shown in the lower-left portion of the screen and we estimated that this "broke presence." The start of the experiment was delayed to recode all instructional and cultural information into an audio mode rather than text, with the hope that audio mode would induce less cognitive overload.

- Recruiting: Originally, we had designed an experiment with 320 subjects and, moreover, we had a design to test a hypothesis that Second Life environments served to motivate subjects to learn non-immersive, web-based, knowledge. This turned out to be difficult since recruiting 


\section{Fishwick, Kamhawi, Coffey and Henderson}

took longer than expected based on student availability. We found that most university students are driven more by extra credit than monetary compensation, but also that extra credit is not a motivation until later on during an academic semester. Due to our need to cover both level of expertise and gender, we encountered difficulty in obtaining needed subjects to fill into the treatment bins, whereas some other treatment bins were full.

In summary, there have been significant obstacles to executing and completing these experiments. In hindsight, we could have simplified the experiments. A simple mechanism would have been to have only one research question and to have chosen fewer variables. However, the complexity and scope of these experiments is expected lead to new insights that go far beyond training transfer questions to where we better understand how, where, and why MUVEs are potentially useful across a wide range of disciplines and training platforms.

\section{ACKNOWLEDGMENTS}

We would like to acknowledge the key research assistants who were essential to the construction of the Second China stimuli and the running of the experiment. For development of the island, Ryan Tanay crafted the artwork and design on the island, as well as doing building construction. Hyungwook Park was responsible for the automated virtual human bots used as helpers, greeters, and guides. For help during the assessment phase of the project, we would like to thank Evan Serge and West Bowers for their significant hours in facilitating the experiment. We would like to thank the U.S. Government, and in particular to the Combating Terrorism Technical Support Office (CTTSO) for funding for the experiments. Special thanks go to Shana Yakobi (CTTSO) and Benjamin Hamilton (SAIC), and to Allison Abbe (U.S. Army Research Institute) for conversations relating to culture and training. The authors would also like to thank Lessiter et al. (2001) for permission to employ their instrument (ITC-SOPI) in our study.

\section{A. EXPERIMENT VARIABLES (DEPENDENT)}

1. Sense of Presence (SOP): engagement, ecological validity/naturalness, negative effects, spatial presence.

2. Subjective Evaluation: sense of being in control/sominnace, emotional valence, emotional arousal, ease of understanding, informativeness of the presentation, importance of presentation, believability of presentation, objectivity of the presentation, believability of the presentation, relevance to the participant.

3. Memory: verbal cued recall (single cue), visual cued recall (single cue), verbal recognition , visual recognition, delayed verbal cued recall (single cue), delayed visual cuded recall (single cue), delayed verbal recognition, delayed visual recognition.

4. Cultural Sensitivity: To Chinese culture ( measured prior, immediately after presentation, and a delayed measure), To Foreign culture (measured prior, immediately after presentation, and a delayed measure).

\section{B. EXPERIMENT VARIABLES (INDEPENDENT)}

1. Immersion: high (through the avatar's eyes), low (over the shoulder).

2. Interaction: high (mouse, keyboard), low (no mouse, keyboard) except for first and last events.

3. Gender: male, female.

4. Expertise: expert, non-expert*.

5. Channel: Second China/MUVE, web page containing animation and text. 


\section{Fishwick, Kamhawi, Coffey and Henderson}

* Expertise is defined as a person (1) with any experience with Second Life, or (2) with any experience with other online virtual environments, including Massively Multiplayer Online Role Playing Games (MMORPGs) such as World of Warcraft, or (3) who spends more than one hour weekly of MMORPGs or more than one hour weekly in an online virtual environment, and (3) who spends more than one hour weekly playing video games in general.

\section{REFERENCES}

Chen, G-M. and W.J. Starosta. 2000. The development and validation of the intercultural sensitivity scale. Paper presented at the Annual Meeting of the National Communication Association, Seattle, Washington. November 8-12, 22 pp.

Cooper, K. 2009. Go with the flow: Engagement and learning in Second Life. In Proceedings of the Interservice/Industry Training, Simulation, and Education Conference (I/ITSEC), paper 9346:1-11.

Dede, C. 2009. Immersive interfaces for engagement and learning. Science, 323: 66-69.

Fishwick, P., ed, 2007. Handbook of dynamic system modeling, CRC Press.

Fishwick, P., J. Henderson, E. Fresh, F. Futterknecht, and B. Hamilton. 2008, Simulating culture: An experiment using a multi-user virtual environment In Proceedings of the 2008 Winter Simulation Conference, eds. S. J. Mason, R. R. Hill, L. Mönch, O. Rose, T. Jefferson, J. W. Fowler, 786-794. Piscataway, New Jersey: Institute of Electrical and Electronics Engineers, Inc.

Geiger, B., and J. Newhagen. 1993. Revealing the black box: Information processing and media effects. Journal of Communication, 43(4), 42-50.

Henderson, J., P.A. Fishwick, E. Fresh, F. Futterknecht, and B. Hamilton. 2008. An Immersive Learning Simulation Environment for Chinese Culture. In Proceedings of the Interservice/Industry Training, Simulation, and Education Conferene (IITSEC), Orlando, Florida, paper 8344, 1-12.

Kamhawi, R. and M.E. Grabe. 2008. Engaging the female audience: An evolutionary psychology perspective on gendered responses to news valence frames. Journal of Broadcasting and Electronic Media. 52(1), 33-51.

Lessiter, J., J. Freeman, E. Keough, and J. Davidoff. 2001. A cross-media presence questionnaire: The ITC- Sense of Presence Inventory, Presence: Teleoperators and Virtual Environments 10(3): 282297.

Luke, S., C. Cioffi-Revilla, L. Panait, and K. Sullivan. 2005. MASON: A multiagent simulation environment, Simulation 81(7): 517-527.

Macredie, R., S. J. E. Taylor, X. Yu, and R. Keeble. 1996. Virtual reality and simulation: An overview. In Proceedings of the 1996 Winter Simulation Conference, ed. J. M. Charnes, D. J. Morrice, D. T. Brunner, and J. J. Swain, 669-674. Piscataway, New Jersey: Institute of Electrical and Electronics Engineers, Inc.

Marsh, T., P. Wright, and S. Smith. 2001. Evaluation for the design of experience in virtual environments: Modeling breakdown of interaction and illusion, CyberPsychology \& Behavior 4(2): 225-238.

Mikropoulos, T. A. 2006. Presence: A unique characteristic in educational virtual environments, Virtual Reality, 10: 197-206.

Monks, T., S. Robinson, and K. Kotiadis. 2009. Model reuse versus model development: Effects on credibility and learning. In Proceedings of the 2009 Winter Simulation Conference, eds. M. D. Rossetti, R. R. Hill, B. Johansson, A. Dunkin and R. G. Ingalls, 767-778. Piscataway, New Jersey: Institute of Electrical and Electronics Engineers, Inc..

Sargent, R. G. 2009. Verification and validation of simulation models. In Proceedings of the 2009 Winter Simulation Conference, eds. M. D. Rossetti, R. R. Hill, B. Johansson, A. Dunkin and R. G. Ingalls, 979-991. Piscataway, New Jersey: Institute of Electrical and Electronics Engineers, Inc.

SC (Second China Project). 2010. Available via $<$ http://www. secondchina.com/> [accessed June 9, 2010]. 
Tako, A. A., and S. Robinson. 2009. Comparing model development in discrete event simulation and system dynamics. In Proceedings of the 2009 Winter Simulation Conference, eds. M. D. Rossetti, R. R. Hill, B. Johansson, A. Dunkin and R. G. Ingalls, 979-991. Piscataway, New Jersey: Institute of Electrical and Electronics Engineers, Inc.

Waterworth, E. and J. Waterworth. 2001. Focus, locus, and sensus: The three dimensions of the virtual experience. Cyberpsychology \& Behavior 4(2): 203-213.

Weber, A., K. Rufer-Bach, and R. Platel. 2008. Creating your world: The official guide to advanced content creation for Second Life. Linden Research, Inc.

Yilmaz, L., T. Ören, and N. G. Aghaee. 2006. Intelligent agents, simulation, and gaming. Simulation \& Gaming, 37(3): 339-349. Sage Publications.

\section{AUTHOR BIOGRAPHIES}

PAUL A. FISHWICK (Ph.D., University of Pennsylvania) is Professor of Computer and Information Science and Engineering at the University of Florida. Fishwick's research interests are in modeling methodology, aesthetic computing, and the use of virtual world technology for modeling and simulation. He is a Fellow of the Society of Modeling and Simulation International, and recently edited the CRC Handbook on Dynamic System Modeling (2007). He served as General Chair of the 2000 Winter Simulation Conference in Orlando, Florida.

RASHA KAMHAWI (Ph.D., Indiana University) is an assistant professor in the Department of Telecommunication at the University of Florida. Her research interests include cognitive and emotional effects of mass media messages on individuals and news media narratives. She has published in Journal of Broadcasting \& Electronic Media, Human Communication Research, Communication Research and Journalism \& Mass Communication Quarterly.

AMY JO COFFEY (Ph.D., University of Georgia) is an assistant professor in the Department of Telecommunication at the University of Florida, where she focuses on audiences and culture, as well as media management issues. Her work has been published in Journalism \& Mass Communication Quarterly, the International Journal on Media Management, and Communication Law \& Policy. She is also a contributing author to the Handbook of Spanish Language Media.

JULIE A. HENDERSON (M. H. Ed., Macquarie University, Sydney, Australia) is the Technology Coordinator at the University of Florida's P.K. Yonge Developmental Research School where she is responsible for the integration of technology into curriculum, professional development focusing on technology, researching emerging technology for education, and managing P.K. Yonge's path to $21^{\text {st }}$ century education. She has done extensive work in online and technology supported education. Her interests include information and communication technologies in education, online immersive learning environments and language and culture. Julie is an active member of the University of Florida's Distance Learning Council and the Technology Innovation Advisory Committee. 